\title{
Si-photonic integrated PZT thin film for acousto-optic modulation
}

\author{
Irfan Ansari ${ }^{1,2,3}$, Tessa Van de Veire ${ }^{2,3}$, John P. George ${ }^{2,3}$, Gilles.F. Feutmba ${ }^{1,2,3}$, Jeroen Beeckman ${ }^{2,3}$, Dries \\ Van Thourhout ${ }^{1,2}$ \\ 1 Photonics Research Group, INTEC Department, Ghent University-IMEC \\ 2 Centre for Nano and Bio-photonics, Ghent University \\ 3 Liquid Crystal \& Photonics Group, ELIS Department, Ghent University \\ Irfan.ansari@ugent.be
}

\begin{abstract}
We demonstrate optical phase modulation in a $\mathrm{Si}$ waveguide by generation of surface acoustic wave using Lead Zirconate Titanate (PZT) thin film. Our measurement shows a $\mathrm{V}_{\pi} \mathrm{L} \approx 3.35$

$\mathrm{V} \mathrm{cm}$ before any device-optimization.
\end{abstract}

\section{Introduction}

Silicon photonics offers a fast-growing technology platform with applications in several important areas such as telecommunication [1], medicine [2], quantum information processing [3] etc. A photonic integrated circuit (PIC) consists of several components like sources, splitters, filters, modulators, detectors etc. Hybrid integration of novel materials is essential to realize these components. In this study, we demonstrate the piezoelectric response of a Siphotonic integrated thin film of Lead Zirconate Titanate (PZT).

PZT is a ferroelectric material with a very high piezoelectric and electromechanical coupling coefficient. However, in most cases PZT deposition involves a Pt-buffer layer to induce preferential crystal growth, which makes it optically lossy. Recently a novel approach for depositing highly textured PZT-films, using a thin transparent lanthanide based buffer layer, was developed. The high quality of the resulting film was proven through the demonstration of efficient electro-optic (EO) modulators on a SiN photonic platform (effective EO coefficient of $\pm 70 \mathrm{pm} / \mathrm{V}$ ) and low optical loss $(1 \mathrm{~dB} / \mathrm{cm})$ [4]. In this work, we exploit the piezoelectric properties of these PZT thin films for acousto-optic modulation.

2. Surface acoustic wave (SAW) excitation with inter-digital transducer (IDT)

A SAW is usually excited by applying an RF signal to an IDT. An IDT facilitates alternate electric fields between the electrodes, which create periodic strain in the piezoelectric material. When an RF signal with SAW resonance frequency is applied to the IDT, a SAW is launched. The wavelength of the primary SAW mode is equal to the period of the IDT.

(a)

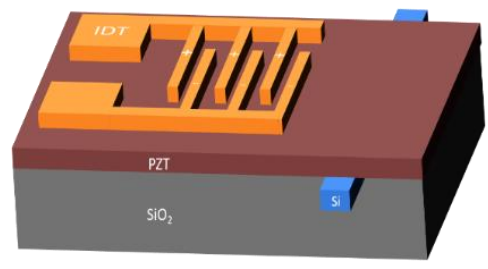

(b)

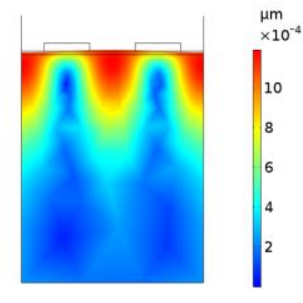

Figure 1: (a) A schematic of the IDTs on PZT/SOI, (b) COMSOL simulation of the electrically driven total displacement (cross-section) for an IDT period on an uniformly in-plane poled PZT layer (200 nm)/SOI.

We use this periodic strain created by the SAW to modulate the optical signal in a waveguide through the photoelastic effect as shown in figure 1(a). The simulation of the SAW as shown in the figure 1(b) suggested that we need to have uniformly in-plane poled PZT for efficient transduction. In order to achieve in-plane poling, we apply a high DC voltage to the IDT. The periodic PZT poling with the IDT causes the SAW frequency to double compared to what is obtained with a uniformly poled layer.

3. Optical phase modulation with SAW

The phase modulation induced by the SAW is given by $\boldsymbol{\phi}=\boldsymbol{\beta} \boldsymbol{\operatorname { s i n }}(\boldsymbol{\Omega} \boldsymbol{t})$, where $\beta$ is the phase modulation amplitude and $\Omega$ is the SAW excitation frequency. In order to characterize this phase modulation, we built a heterodyne setup as shown in figure 2(a). The output power from the photodetector (PD) is given by the following equation: 
(1)

$$
\mathrm{P}_{\text {out }} \propto A 1 * A 2\left[\cos \Delta \omega t+\frac{\beta}{2}(\cos (\Omega-\Delta \omega) t-\cos (\Omega+\Delta \omega) t)\right]
$$

where A1 and A2 are the amplitude of the optical signals coming through the DUT and the acousto-optic modulator (AOM) respectively. The signal from the AOM is frequency shifted by $\Delta \omega=200 \mathrm{MHz}$. Thus, the electrical spectrum analyzer (ESA) shows two sidebands at frequencies $\Omega_{\mathrm{SAW}}+\omega_{\mathrm{AOM}}$ and $\left|\Omega_{\mathrm{SAW}}-\omega_{\mathrm{AOM}}\right|$ and a peak signal at the AOM as shown in figure 2(b). We observe that the SAW resonance peak is consistent with the IDT period as shown in figure 2(c). The additional peaks are obtained due to the excitation of the bulk modes. The SAW resonance frequency at 576 $\mathrm{MHz}$ (for the IDT period $12 \mu \mathrm{m}$ ) is further confirmed with the electrical S11 measurement as shown in the figure 2(d). Therefore, both optical and electrical experiments prove the SAW excitation.

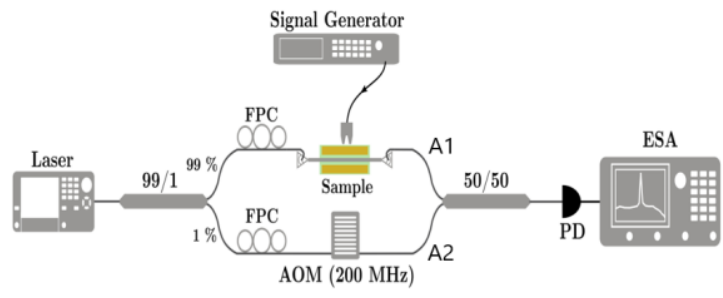

(a)

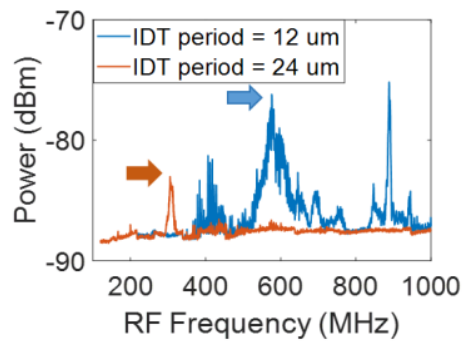

(c)
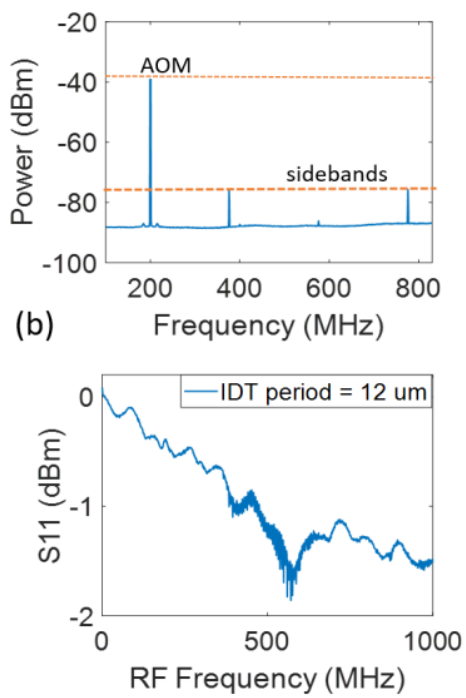

(d)

Figure 2: (a) A schematic of the heterodyne setup to measure the phase modulation in a waveguide, (b) the sidebands from the phase modulation for IDT period $12 \mu \mathrm{m}$ with RF power of $15 \mathrm{dBm},(\mathrm{c})$ the modulated peak signal is maximum at a certain frequency when SAW is excited, and the SAW frequency is consistent with the IDT period, (d) the electrical S11 parameter for the IDT period $12 \mu \mathrm{m}$ show dip at $576 \mathrm{MHz}$.

From the ratio of the AOM and sideband peaks, we calculate the phase modulation amplitude $(\beta)$ to be 0.03 radians for the IDT with period $12 \mu \mathrm{m}$ (finger-width $3 \mu \mathrm{m}$ ), which corresponds to $\mathrm{V}_{\pi} \mathrm{L} \approx 3.35 \mathrm{~V} \mathrm{~cm}$. For a similar PZT layer, earlier the electro-optic modulator has been reported to give $\mathrm{V}_{\pi} \mathrm{L} \approx 3.2 \mathrm{~V} \mathrm{~cm}$ [4]. Furthermore, by sweeping the $\mathrm{RF}$ drive power on IDT, we obtain the modulation efficiency $\left(\Delta \mathrm{n}_{\text {eff }} / \Delta \mathrm{V}\right)$ of $2.29 \times 10^{-5} \mathrm{~V}^{-1}$. Hence, we notice that even without any optimization, our first preliminary results already give competitive figures of merit.

\section{Conclusion}

We have demonstrated phase modulation in a WG circuit induced by SAW. The figures of merit for this PZT based acousto-optic modulator are already competitive to the existing state-of-the-art modulators. Further optimization such as impedance matching etc. is expected to enhance the device performance. The piezoelectric response of this Siphotonic integrated PZT film opens the possibility for MEMS based photonic integrated devices such as tunable filters, on-chip acousto-optic modulators etc.

\section{References}

[1]Doerr CR, "Silicon photonic integration in telecommunications". Front. Phys. 3:37 (2015).

[2]Estevez, M. C., Alvarez, M., \& Lechuga, L. M, "Integrated optical devices for lab-on-a-chip biosensing applications. Laser \& Photonics Reviews", 6(4), 463-487 (2012).

[3] Wang, J., Sciarrino, F., Laing, A. et al., “Integrated photonic quantum technologies”, Nature Photonics (2019)

[4] K. Alexander et al, "Nanophotonic Pockels modulators on a silicon nitride platform", Nature Communications volume 9, Article number: 3444 (2018).

\footnotetext{
**Acknowledgment: This work was supported by the EU commission through Grant agreement No. 732894 (FET proactive HOT).
} 